\title{
CARACTERIZAÇÃO FÍSICA, QUÍMICA E NUTRICIONAL DA PITAIA-ROSA DE POLPA VERMELHA ${ }^{1}$
}

\author{
MARIA HELENA MENEZES CORDEIRO², JUCELIANDY MENDES DA SILVA ${ }^{3}$, \\ GISELE POLETE MIZOBUTSI ${ }^{4}$, EDSON HIYDU MIZOBUTSI ${ }^{4}$, WAGNER FERREIRA DA MOTA ${ }^{4}$
}

RESUMO- A pitaia-rosa de polpa vermelha é uma cactácea com potencial para exploração econômica, devido aos seus componentes nutricionais e ao alto valor comercial; no entanto, poucas são as pesquisas quanto a suas características pós-colheita. O objetivo do trabalho foi caracterizar física, química e nutricionalmente pitaias-rosas de polpa vermelha. Frutos colhidos totalmente maduros foram sanitizados, selecionados e divididos em grupos de quatro frutos por repetição, constituindo um total de 30 amostras. Em seguida, as amostras foram submetidas às seguintes avaliações: comprimento, diâmetro, espessura de casca, massa fresca, massa de casca e polpa, teor de umidade, firmeza da casca, sólidos solúveis, $\mathrm{pH}$, acidez titulável, açúcares e fibra bruta, cromaticidade, ângulo hue e luminosidade da casca e da polpa, e o teor de nutrientes. Para os dados obtidos, foram determinados a média, o valor mínimo e máximo, desvio-padrão e o coeficiente de variação. A fruta apresentou valores elevados de conteúdo de água, teor de fibras e dos nutrientes nitrogênio, potássio, cálcio, ferro, manganês e zinco. Quanto ao teor de sólidos solúveis, a média observada foi de $13,14^{\circ}$ Brix, e para acidez titulável,0,29 mg de ác. málico $100^{-1} \mathrm{~mL}$ de suco. Verificaram-se para os açúcares valores de 5,56; 8,79 e 3,07 \%, respectivamente, para os açúcares redutores, totais e não redutores. Quanto à coloração, os frutos apresentaram valores maiores para luminosidade e cromaticidade da casca e ângulo Hue da polpa. A pitaia-rosa de polpa vermelha (Hylocereus polyrhizus) apresenta características físicas, químicas e nutricionais interessantes para o consumo in natura.

Termos para indexação: Hylocereus polyrhizus, frutas exóticas, cactáceas.

\section{PHYSICAL, CHEMICAL AND NUTRITIONAL CHARACTERIZATION OF PINK PITAYA OF RED PULP}

\begin{abstract}
The pink pitaya of red pulp is a cactacea with potential for economic exploitation, due to its nutritional components and high commercial value, however there is little research about its post-harvest characteristics. The aim of this study was to characterize physical, chemical and nutritionally pink pitaya of red pulp. Fruits harvested fully ripe were sanitized, selected and divided into groups of four fruits per repetition, constituting a total of 30 samples. Then the samples were subjected to the following evaluations: length, diameter, peel thickness, fresh matter, peel and pulp mass, moisture content, peel firmness, soluble solids, $\mathrm{pH}$, titratable acidity, sugars and crude fiber, chromaticity, hue angle and brightness of the peel and pulp and nutrient content. For the obtained data, it was determined mean, minimum and maximum values, standard deviation and coefficient of variation. The fruit showed high water content, content of fiber and nutrients nitrogen, potassium, calcium, iron, manganese and zinc. As for soluble solids content it was observed average of $13.14^{\circ}$ Brix and titratable acidity $0.29 \mathrm{mg}$ of Malic acid in $100^{-1} \mathrm{ml}$ of juice. For sugars were found values of 5.56, 8.79 and $3.07 \%$ respectively for the reducing sugars, total and non-reducing ones. As for color, the fruits showed higher values for brightness and chromaticity of the peel and the Hue angle of the pulp. The pink pitaya of red pulp (Hylocereus polyrhizus) has interesting physical, chemical and nutritional characteristics to be consumed in nature.
\end{abstract}

Index terms: Hylocereus polyrhizus, exotic fruits, cactaceae.

\footnotetext{
'(Trabalho 046-14). Recebido em: 12-02-2014. Aceito para publicação em: 05-08-2014.

${ }^{2}$ Eng $^{\mathrm{a}}$.Agr ${ }^{\mathrm{a}}$, Doutoranda em Fitotecnia, UFV. Laboratório de Fisiologia Pós-colheita, (38) 3821 2756. Avenida P.H. Rolfs, s/n - Campus Universitário, Viçosa, MG, 36571-000. E-mail; helenaagro@yahoo.com.br.

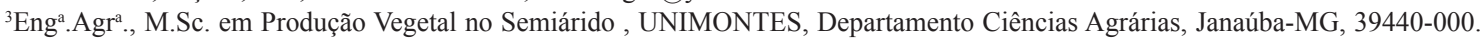
E-mail: juceliandy@yahoo.com.br;

${ }^{4}$ Eng $^{\circ}$.Agr ${ }^{\circ}$., D.Sc. Prof. da Universidade Estadual de Montes Claros, Departamento Ciências Agrárias, Janaúba-MG, 39440-000. E-mails: gisele.mizobutsi@unimontes.br, edson.mizobutsi@unimontes.br,wagner.mota@unimontes.br.
} 


\section{INTRODUÇÃO}

A pitaia-rosa de polpa vermelha também chamada apenas de 'pitaia-vermelha' é uma fruta tropical que apresenta aceitação crescente nos mercados consumidores. Sua aparência exótica, sabor doce e suave, polpa firme e suas características nutricionais e funcionais tornam seu cultivo considerado promissor (MARQUES et al., 2011).

A pitaia é uma planta rústica da família Cactaceae, conhecida como "Dragon Fruit (Frutado-Dragão)". São encontradas diferentes espécies de pitaia, sendo algumas comerciais e outras nativas. De acordo com a espécie, seus frutos podem apresentar características físicas e químicas diversificadas quanto ao formato, presença de espinhos, cor da casca e da polpa, teor de sólidos solúveis e $\mathrm{pH}$ na polpa, reflexo da alta diversidade genética desta frutífera (LIMA et al., 2013).

Do gênero Hylocereus, a pitaia é originária das Américas e encontra-se distribuída em diversos países do continente. Apresenta potencial agronômico e econômico, devido sua rusticidade (ORTIZ-HERNANDEZ et al., 1999). Dentro do gênero Hylocereus, encontram-se as espécies mais cultivadas: $H$. undatus, $H$. polyrhizus e $H$. costaricensis. Seus frutos apresentam superfície escamosa, sendo que $H$. undatus apresenta superfície rosa e polpa branca e $H$. polyrhizus superfície rosa e polpa vermelha (FERNANDES et al., 2010). Não há registro no País de nenhuma variedade de pitaya recomendada, as mudas comercializadas não são de material selecionado, verificando-se variação na produção, tamanho, formato e características físico-químicas dos frutos (LIMA et al., 2013; JUNQUEIRA et al., 2010). Segundo Silva et al. (2011), com a abertura comercial, o mercado mundial de frutas tem-se tornado mais competitivo e aberto às novidades, como frutas nativas e exóticas,principalmente devido às divulgações da mídia sobre os benefícios do consumo de frutas, destacando-as como alimento saudável, balanceado, funcional e diversificado, com suas cores, formatos, cheiros e sabores, o que despertou nos consumidores o desejo por frutas nativas e exóticas.

As pitaias encontram-se dentro do grupo de frutas exóticas e são fontes de vitaminas e minerais, beneficiando-se do interesse do mercado consumidor. Por apresentarem alto valor comercial, elas despertam o interesse dos fruticultores em seu plantio e cultivo. Na região Sudeste, a produção de frutos ocorre principalmente nos meses de dezembro a maio, e a produtividade média é de 14 toneladas de frutos ha-1 (BASTOS et al., 2006).

Diante da possibilidade de cada vez mais explorar economicamente as pitaias devido ao seu alto valor econômico e sua rusticidade, adaptando-se às condições desfavoráveis, como o déficit hídrico, faz-se necessário um estudo detalhado de suas características físicas, químicas e nutricionais. Com isso, o objetivo do presente trabalho foi caracterizar física, química e nutricionalmente pitaias-rosas de polpa vermelha (Hylocereus polyrhizus).

\section{MATERIAL E MÉTODOS}

As pitaias-rosa de polpa vermelha foram colhidas completamente maduras, em uma propriedade na zona rural de Janaúba-MG, à altitude de $544 \mathrm{~m}$, situado a $15^{\circ} 49^{\prime} 48^{\prime \prime}$ de latitude sul e $43^{\circ} 16^{\prime} 08^{\prime \prime}$ de longitude oeste, em um pomar irrigado, cultivado sobre Latossolo VermelhoAmarelo. As plantas com cerca de um ano de idade foram adubadas apenas com esterco bovino (14 kg planta $^{-1}$ ), não sendo realizada adubação química, pois não há recomendação de adubação para a cultura. $\mathrm{O}$ período de floração ocorreu entre janeiro e fevereiro, e os frutos foram coletados de uma única vez, na segunda semana de março.

Os frutos foram lavados e selecionados, sendo descartados os que apresentavam algum tipo de injúria. Posteriormente, foram divididos em grupos de quatro, constituindo-se ao final 30 amostras, com quatro frutos cada uma.

A partir das amostras de quatro frutos, foram obtidas as médias das seguintes características: a) comprimento $(\mathrm{cm})$, diâmetro $(\mathrm{cm})$ e espessura de casca $(\mathrm{mm})$, obtidas através de paquímetro; b) massa fresca total, da casca e da polpa (g), medida por meio de balança digital de precisão; c) teor de umidade $\left(\mathrm{g} 100^{-1} \mathrm{~g}\right)$, feita em estufa a $105^{\circ} \mathrm{C}$ até peso constante (INSTITUTO ADOLFO LUTZ, 2008); d) firmeza da casca $(\mathrm{N})$, determinada pelo uso de penetrômetro digital com ponteira de $0,8 \mathrm{~cm}$ de diâmetro; e) sólidos solúveis ( ${ }^{\circ}$ Brix), obtido por meio de refratometria, utilizando-se de um refratômetro digital; f) pH, medido em pHmetro digital PG1800; g) acidez titulável (mg de ác. málico $100 \mathrm{~mL}^{-1}$ de suco), determinado por meio da titulação de 10 $\mathrm{mL}$ de suco homogeneizado com $90 \mathrm{~mL}$ de água destilada, utilizando-se como titulante de solução de $\mathrm{NAOH} 0,1 \mathrm{~N}$, adicionando à amostra três gotas de fenolftaleína a $1 \%$ como indicador; $h$ ) açúcares redutores $(\%)$, obtido por espectrofotometria, utilizando-se de espectrofotômetro com leitura a 510 $\mathrm{nm}$, segundo o método descrito por Nelson (1944); h) açúcares totais (\%), realizado por espectrofotometria, 
utilizando-se de espectrofotômetro com leitura a 620 nm, segundo o método descrito por Dische (1962); i) açúcares não redutores (\%),determinado pela diferença dos açúcares totais e açúcares redutores; j) a fibra bruta (\%), foi realizada submetendo as amostras à digestão ácida, com solução de ácido sulfúrico a $1,25 \%$, seguida por digestão alcalina com hidróxido de sódio a 1,25\% (BRASIL, 2005), e k) coloração da casca e polpa, obtida por meio de colorímetro de bancada com leitura direta de reflectância das coordenadas $\mathrm{L}^{*}$ (luminosidade), $\mathrm{a}^{*}$ (tonalidade vermelha ou verde) e $\mathrm{b}^{*}$ (tonalidade amarela ou azul), do sistema Hunterlab Universal Software. Foi obtida diretamente a luminosidade $\left(\mathrm{L}^{*}\right)$, e a partir dos valores de $\mathrm{a}^{*} \mathrm{e} \mathrm{b}^{*}$, calcularam-se o ângulo hue $\left({ }^{\circ} h^{*}\right)$ ( A1 e A2) e o índice de saturação croma $\left(\mathrm{C}^{*}\right)(\mathrm{B})$.

${ }^{\circ} \mathrm{h}^{*}=\operatorname{actg}\left(\mathrm{a}^{*} / \mathrm{b}^{*}\right)(-1)+90 \rightarrow$ para $\mathrm{a}^{*}$ negativo $(\mathrm{A} 1)$

${ }^{\circ} \mathrm{h}^{*}=90-\left(\operatorname{actg}\left(\mathrm{a}^{*} / \mathrm{b}^{*}\right)\right) \rightarrow$ para $\mathrm{a}^{*}$ positivo

$C^{*}=\sqrt{ }\left(a^{*}\right)^{2}+\left(b^{*}\right)^{2}$

Os teores de nutrientes nas pitaias-rosa de polpa vermelha foram obtidos por meio do extrato nítrico-perclórico. Os teores de $\mathrm{P}$ foram determinados por colorimetria; $\mathrm{Ca}, \mathrm{Mg}, \mathrm{Cu}, \mathrm{Fe}, \mathrm{Mn}$ e $\mathrm{Zn}$, por espectrofotometria de absorção atômica, e K por fotometria de chama. Os teores de $\mathrm{N}$ total foram determinados pelo método semimicro Kjeldahl. O $\mathrm{B}$, após digestão por via seca, foi determinado por colorimetria (método da curcumina) (MALAVOLTA et al., 1997).

Para os dados obtidos, foram determinadas as médias, o valor mínimo e máximo, o desvio-padrão e o coeficiente de variação, por meio do programa

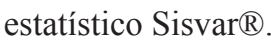

\section{RESULTADOS E DISCUSSÃO}

As pitaias-rosa, de polpa vermelha $(H$. polyrhizus) apresentaram valores médios para comprimento e diâmetro de fruto de, respectivamente, 10,73 e $8,07 \mathrm{~cm}$ (Tabela 1). Na literatura, são observados valores médios de $8,5 \mathrm{~cm}$ de comprimento e 7,3 cm de diâmetro (ESQUIVEL et al., 2007; MOREIRA et al.,2011). Estes valores são inferiores aos verificados neste experimento, provavelmente resultado das diferenças edafoclimáticas da região norte de Minas Gerais, em comparação aos cultivos na região de sul de Minas Gerais e países da américa central. Para a espessura da casca, verificou-se valor médio de 2,9 mm, enquanto para a massa da casca foi observada uma média de 111,23 g (Tabela 1), representando próximo de $27 \%$ da massa total dos frutos. Estes resultados são superiores aos verificados por Yah et al. (2008), que observaram que 20,40\% do peso dos frutos de pitaia-vermelha correspondem ao peso da casca. Quanto à massa fresca dos frutos e massa da polpa de pitaia-rosa de polpa vermelha, verificaram-se médias de 411, 22 g e 309,44 g, respectivamente. Observa-se que a pitaia apresenta uma grande quantidade de polpa quando comparada a outras cactáceas, e esta característica pode ser interessante tanto para o consumo in natura como para o processamento do produto.

A firmeza da casca da pitaia-rosa da polpa vermelha, conforme a Tabela 1, apresentou média de $44,31 \mathrm{~N}$, enquanto o teor de umidade verificado foi $87,14 \mathrm{~g} 100^{-1} \mathrm{~g}$, indicando que o fruto apresenta alta concentração de líquidos. Yah et al. (2008), estudando as características de pitaias-vermelhas de polpa branca durante seu desenvolvimento, verificaram que frutos colhidos 31 dias depois da antese apresentaram valores de firmeza da polpa de 6,3 N. Wall e Khan (2008) observaram valores de firmeza da polpa de 5,7 $\mathrm{N}$ para frutos de pitaiavermelha de polpa branca colhidos aos 57 dias após a antese, totalmente maduros.

As pitaias apresentaram valores de sólidos solúveis, $\mathrm{pH}$ e acidez titulável, respectivamente, de $13,14{ }^{\circ}$ Brix, 5,60; 0,29 mg de àc. málico $100^{-1} \mathrm{~mL}$ de suco (Tabela 2). Enciso et al. (2011) encontraram para frutos de pitaia, $H$. undatus, colhidos totalmente maduros, valores de sólidos solúveis entre 11,6 e 13,6 ${ }^{\circ}$ Brix e de acidez de 0,63 mg de àc. málico, valores próximos aos encontrados neste trabalho para $H$. polyrhizus. Yah et al. (2008), trabalhando com frutos de pitaia-vermelha de polpa branca, observaram valores de sólidos solúveis de $12,6^{\circ}$ Brix de acidez de 0,40 mg de ác. málico. Estes resultados indicam que, apesar de serem de espécies diferentes, os valores de sólidos solúveis e acidez titulável são semelhantes, com isso a aceitabilidade de $H$. polyrhizus pelo consumidor pode ser favorecida.

Quanto aos açúcares totais, redutores e não redutores, foram observados valores médios de 8,79; 5,56 e 3,07\%\%. Os açúcares constituem a maior parte dos sólidos solúveis em forma de glicose, frutose e sacarose. Segundo Santos et al. (2010), frutos com altos teores de sólidos solúveis são preferidos tanto para consumo in natura quanto para a industrialização, por propiciarem maior rendimento no processamento, em razão da maior quantidade de néctar produzida por quantidade de polpa. Observa-se que a pitaia apresenta valores baixos de açúcares e pouca acidez, sendo mais indicada para 
o consumo in natura. A sacarose é encontrada em menores quantidades em frutos de Hylocereus spp. compreendendo cerca de $2 \%$ do açúcar total (WALL; KHAN, 2008).

As pitaias- rosas de polpa vermelha apresentaram teores de fibra bruta elevados. O teor médio de fibra bruta observado foi de 11,35\%. As fibras alimentares são partes dos frutos que se apresentam resistentes à digestão e à absorção no intestino delgado de humanos, sendo importantes por reduzir o risco de certas doenças. A Food and Drug Administration (FDA) recomenda o consumo de 25 $\mathrm{g}$ de fibra alimentar total por dia em uma dieta de 2.000 calorias (FREIRE et al., 2012). Nesse sentido, o consumo de $100 \mathrm{~g}$ do fruto in natura fornece cerca de $11,35 \mathrm{~g}$ do que é recomendado.

Para a coloração da casca, verificou-se para a cromaticidade, ângulo Hue e luminosidade valores médios de 42,$72 ; 15,73^{\circ}$ e 42,41 , respectivamente (Tabela 3) . Chik et al. (2011) encontraram valores de cromaticidade e ângulo hue, respectivamente, de 40 e $10,70^{\circ}$ e Phebe et al. (2009) verificaram valores de 40,25 para luminosidade da casca, ambos os autores estudando $H$. polyrhizus produzidas na Malásia. Estes valores são semelhantes aos verificados neste trabalho, porém o ângulo hue e a luminosidade apresentaram-se inferiores, indicando uma coloração mais próxima do rosa, provavelmente devido a efeitos das condições ambientais que são distintas.

Quanto à coloração da polpa, foram observados valores de 38,$89 ; 172,00^{\circ}$ e 14,25 de cromaticidade, ângulo hue e Luminosidade, respectivamente (Tabela 3). Os valores de cromaticidade, ângulo hue da polpa apresentaram-se extremamente superiores aos observados por Chik et al. (2011), com valores respectivos de 27,8 e $3,10^{\circ}$ para $H$. polyrhizus. Comparando-se estes resultados aos encontrados neste experimento, verifica-se que as pitaias produzidas no norte de Minas Gerais apresentam cor intermediaria entre vermelho e amarelo, enquanto as frutas da Malásia apresentam coloração propriamente vermelha. Quanto à luminosidade da polpa, Phebe et al. (2009) verificaram para $H$. polyrhizus valor de 33,75 , resultado superior ao observado no presente trabalho, indicando que as pitaias de polpa vermelha produzidas no norte de Minas apresentam menor brilho. Este resultado pode ter sido influenciado pelas diferenças observadas para cromaticidade e ângulo hue com relação à literatura citada.

Os frutos apresentaram em sua composição maior concentração dos macronutrientes nitrogênio, potássio e cálcio, apresentando médias, respectivamente, de 11,3; 12,6 e 8,0 $\mathrm{g} \mathrm{kg}^{-1}$ (Tabela 4). Os macronutrientes fósforo, enxofre e magnésio foram verificados nos frutos, nas concentrações de 2,3; 1,0 e 3,6 $\mathrm{g} \mathrm{kg}^{-1}$. Quanto aos micronutrientes analisados, verificou-se que o ferro, o zinco e o manganês se encontram nas maiores concentrações. Os teores médios de ferro, zinco e manganês foram, respectivamente, de 337,58; 116,26 e 113,93 mg kg${ }^{1}$. Os micronutrientes nas menores concentrações no fruto são o boro e o cobre, que apresentaram valores médios de 18,73 e $21,71 \mathrm{mg} \mathrm{kg}^{-1}$, respectivamente.

A pitaia-rosa de polpa vermelha apresenta teores significativos de minerais importantes na dieta humana. Além disso, o teor elevado de cálcio pode indicar maior resistência dos frutos após a colheita. Segundo Natale et al. (2005), em frutíferas, o cálcio desempenha papel importante, pois afeta a qualidade do fruto e sua capacidade de armazenamento depois da colheita. Existe relação direta entre o conteúdo de cálcio nos frutos e o amolecimento, firmeza e tempo de vida útil de prateleira. 
TABELA 1- Valores médios com seus respectivos valores mínimos, máximos, desvios- padrão $(\sigma)$ e coeficientes de variação (CV) das características comprimento, diâmetro, espessura de casca, massa fresca, massa da casca, massa da polpa, teor de umidade e firmeza da casca de pitaias -rosas de polpa vermelha (Hylocereus polyrhizus).

\begin{tabular}{cccccc}
\hline Determinações & Média & Mínimos & Máximos & $\boldsymbol{\sigma}$ & CV (\%) \\
\hline Comprimento $(\mathrm{mm})$ & 107,06 & 102,00 & 111,00 & 3,89 & 3,63 \\
Diâmetro (mm) & 84,46 & 82,5 & 87,00 & 1,63 & 1,93 \\
Espessura de casca $(\mathrm{mm})$ & 2,90 & 2,50 & 3,00 & 0,17 & 6,05 \\
Massa fresca total $(\mathrm{g})$ & 411,22 & 385,05 & 420,22 & 8,95 & 2,17 \\
Massa da casca $(\mathrm{g})$ & 111,23 & 95,93 & 123,20 & 8,82 & 7,93 \\
Massa da polpa $(\mathrm{g})$ & 309,44 & 268,89 & 338,23 & 24,72 & 7,99 \\
Teor de umidade $\left(\mathrm{g} 100^{-1} \mathrm{~g}\right)$ & 87,14 & 85,62 & 88,89 & 1,58 & 1,81 \\
Firmeza $(\mathrm{N})$ & 44,31 & 41,16 & 46,55 & 2,01 & 4,54 \\
\hline
\end{tabular}

$\boldsymbol{\sigma}:$ desvio- padrão, CV: coeficiente de variação, *: Valores médios obtidos a partir da média de 30 amostras de quatro frutos cada.

TABELA 2- Valores médios* com seus respectivos valores mínimos, máximos, desvios-padrão $(\sigma)$ e coeficientes de variação $(\mathrm{CV})$ das características sólidos solúveis, $\mathrm{pH}$, acidez titulável e porcentagem de açúcar redutor, total, não redutor e fibra bruta de pitaia-rosa de polpa vermelha (Hylocereus polyrhizus).

\begin{tabular}{cccccc}
\hline Determinações & Média & Mínimos & Máximos & $\boldsymbol{\sigma}$ & CV (\%) \\
\hline Sólidos solúveis ( ${ }^{\circ}$ Brix) & 13,14 & 12,10 & 13,80 & 0,56 & 4,32 \\
pH & 5,32 & 5,09 & 5,60 & 0,21 & 3,87 \\
Acidez titulável ( mg de àc. málico $100^{-1} \mathrm{~mL} \mathrm{de} \mathrm{suco)}$ & 0,29 & 0,26 & 0,35 & 0,30 & 10,27 \\
Açúcar redutor (\%) & 5,56 & 4,87 & 6,05 & 0,37 & 6,63 \\
Açúcar total (\%) & 8,79 & 8,07 & 9,61 & 0,59 & 6,79 \\
Açúcar não redutor (\%) & 3,07 & 2,82 & 3,38 & 0,2 & 6,54 \\
Fibra bruta (\%) & 11,35 & 10,41 & 11,97 & 0,57 & 5,01 \\
\hline
\end{tabular}

б: desvio-padrão, CV: coeficiente de variação, *: Valores médios obtidos a partir da média de 30 amostras de quatro frutos cada.

TABELA 3- Valores médios* com seus respectivos valores mínimos, máximos, desvios- padrão $(\sigma)$ e coeficientes de variação $(\mathrm{CV})$ das características descritoras da cor, cromaticidade, ângulo Hue e luminosidade da casca e da polpa de frutos de pitaia- rosa de polpa vermelha (Hylocereus polyrhizus).

\begin{tabular}{cccccc}
\hline Determinações & Média & Mínimos & Máximos & $\boldsymbol{\sigma}$ & CV (\%) \\
\hline Cromaticidade da casca & 42,75 & 41,54 & 44,42 & 0,98 & 2,30 \\
Cromaticidade da polpa & 38,89 & 38,08 & 40,04 & 0,61 & 1,56 \\
Ângulo hue da casca $\left(^{\circ}\right)$ & 15,73 & 13,72 & 17,79 & 1,44 & 9,16 \\
Ângulo hue da polpa $\left(^{\circ}\right)$ & 172,00 & 164,47 & 177,85 & 4,45 & 2,58 \\
Luminosidade da casca & 42,41 & 41,61 & 43,37 & 0,58 & 1,38 \\
Luminosidade da polpa & 14,25 & 12,83 & 15,58 & 0,95 & 6,71 \\
\hline
\end{tabular}

$\boldsymbol{\sigma}$ : desvio-padrão, CV: coeficiente de variação, *: Valores médios obtidos a partir da média de 30 amostras de quatro frutos cada. 
TABELA 4- Valores médios* com seus respectivos valores mínimos, máximos, desvios- padrão $(\sigma)$ e coeficientes de variação $(\mathrm{CV})$ para os nutrientes nitrogênio $(\mathrm{N})$, fósforo $(\mathrm{P})$, potássio $(\mathrm{K})$, enxofre $(\mathrm{S})$, cálcio $(\mathrm{CA})$, magnésio $(\mathrm{Mg})$, boro $(\mathrm{B})$, cobre $(\mathrm{CU})$, ferro $(\mathrm{Fe})$, manganês $(\mathrm{Mn})$ e zinco $(\mathrm{Zn})$ de frutos de pitaia -rosa de polpa vermelha (Hylocereus polyrhizus).

\begin{tabular}{cccccc}
\hline Determinações & Média & Mínimos & Máximos & $\boldsymbol{\sigma}$ & CV (\%) \\
\hline $\mathrm{N}\left(\mathrm{g} \mathrm{Kg}^{-1}\right)$ & 11,3 & 10,6 & 12,9 & 0,10 & 9,6 \\
$\mathrm{P}\left(\mathrm{g} \mathrm{Kg}^{-1}\right)$ & 2,3 & 2,0 & 2,7 & 0,03 & 12,74 \\
$\mathrm{~K}\left(\mathrm{~g} \mathrm{Kg}^{-1}\right)$ & 12,6 & 11,8 & 13,3 & 0,06 & 5,19 \\
$\mathrm{~S}\left(\mathrm{~g} \mathrm{Kg}^{-1}\right)$ & 1,0 & 0,9 & 1,1 & 0,01 & 7,43 \\
$\mathrm{Ca}\left(\mathrm{g} \mathrm{Kg}^{-1}\right)$ & 8,0 & 7,4 & 8,8 & 0,05 & 6,64 \\
$\mathrm{Mg}\left(\mathrm{g} \mathrm{Kg}^{-1}\right)$ & 3,6 & 3,4 & 4,0 & 0,02 & 7,61 \\
$\mathrm{~B}\left(\mathrm{mg} \mathrm{Kg}^{-1}\right)$ & 18,73 & 17,69 & 19,91 & 1,02 & 5,49 \\
$\mathrm{Cu}\left(\mathrm{mg} \mathrm{Kg}^{-1}\right)$ & 21,71 & 19,1 & 24,00 & 1,87 & 8,62 \\
$\mathrm{Fe}\left(\mathrm{mg} \mathrm{Kg}^{-1}\right)$ & 337,58 & 307,50 & 360,00 & 21,10 & 6,25 \\
$\mathrm{Mn}\left(\mathrm{mg} \mathrm{Kg}^{-1}\right)$ & 113,93 & 94,8 & 138,30 & 15,82 & 13,89 \\
$\mathrm{Zn}\left(\mathrm{mg} \mathrm{Kg}^{-1}\right)$ & 116,26 & 114,00 & 118,40 & 1,65 & 1,42 \\
\hline
\end{tabular}

$\boldsymbol{\sigma}$ : desvio- padrão, CV: coeficiente de variação, *: Valores médios obtidos a partir da média de 30 amostras de quatro frutos cada.

\section{CONCLUSÃO}

A pitaia-rosa de polpa vermelha $(H$. polyrhizus) apresentou características físicas, químicas e nutricionais aceitáveis para o consumo in natura, destacando-se o alto valor de firmeza da casca, baixo teor de acidez e o teor de sólidos solúveis próximo ao observado para outras espécies do gênero, além de valores significativos de fibras e minerais, como o cálcio,o potássio e o ferro.

\section{AGRADECIMENTOS}

Os autores agradecem pelo apoio financeiro da CAPES e FAPEMIG.

\section{REFERÊNCIAS}

BASTOS, D.C.; PIO, R.; SCARPARE FILHO, J.A.; LIBARDI, M.N.; ALMEIDA, L.F.P.; GALUCHI, T.P.D.; BAKKER, S.T. Propagação da Pitaya'vermelha' por estaquia. Ciência e Agrotecnologia, Lavras, v.30, n.6, p.1106-1109, 2006.

BRASIL. Ministério da Saúde. Agência Nacional de Vigilância Sanitária. Métodos físico-químicos para análise de alimentos. Brasília: Ministério da Saúde, 2005. 1018p.
CHIK, C. T.; BACHOK, S.; BABA, N. Quality characteristics and acceptability of three types of pitaya fruits in a consumer acceptance test. Journal of Tourism, Hospitality \& Culinary Arts, Shah Alam v. 3, n. 1, 89-98, 2011.

DISCHE, Z. General color reactions. In: WHISTLER, R. L.; WOLFRAM, M. L. Carbohydrate chemistry. New York: Academic Press, 1962. p. 477-512.

ENCISO, T. O.; ZAZUETA, M. E. I.; M. RANGEL, M. D. M.; TORRES, J. B. V.; ROMERO, M. V.; VERDUGO, S. H. Calidad postcosecha de frutos de pitahaya (Hylocereus undatus haw.) cosechados en tres estados de madurez. Revista Fitotecnia Mexico, Chapingo, v. 34, n. 1, p. 63-72, 2011.

ESQUIVEL, P.; STINTZING, F. C.; CARLE, R. Comparison of morphological and chemical fruit traits from different pitaya genotypes (Hylocereus sp.) grown in Costa Rica. Journal of Applied Botany and Food Quality, San Pedro, v. 81, p.714, 2007.

FERNANDES, L. M. S.; VIEITES, R. L.; CERQUEIRA, R. C.; BRAGA, C. L.; SIRTOLI, L. F.; AMARAL, J. L. Características pós-colheita em frutos de pitaya orgânica submetida a diferentes doses de irradiação. Revista Biodiversidade, Rondonópolis, v. 9, n. 1, p. 15-22, 2010. 
FREIRE, J. M.; ABREU, C. M. P.; CORRÊA, A. D.; SIMÂO, A. A.;SANTOS, C. M. Avaliação de compostos funcionais e atividade Antioxidante em farinhas de polpa de goiabas. Revista Brasileira de Fruticultura, Jaboticabal, v. 34, n. 3, p. 847-852, 2012.

INSTITUTO ADOLFO LUTZ. Normas Analíticas do Instituto Adolfo Lutz: métodos químicos e físicos para análise de alimentos. 3. ed. São Paulo: IMESP, 2008. v.1, p. 98-99.

JUNQUEIRA, K. P.; FALEIRO, F. G.; BELLON, G.; JUNQUEIRA, N. T. V.; FONSECA, K. G.; LIMA, C. A.; SANTOS, E. C. Variabilidade genética de acessos de pitaia com diferentes níveis de produção por meio de marcadores RAPD. Revista Brasileira de Fruticultura, Jaboticabal, v. 32, n. 3, p. 840-846, 2010.

LIMA, C. A.; FALEIRO, F. G.; JUNQUEIRA, N. T. V. Diversidade genética intra e interespecífica de pitaia com base nas características físico-químicas de frutos. Revista Brasileira de Fruticultura, Jaboticabal, v. 35, n. 4, p. 1066-1072, 2013.

LIMA, C. A.; FALEIRO, F. G.; JUNQUEIRA, N. T. V.; COHEN, K. O.; GUIMARÃES, T. G. Características físico-químicas, polifenóis e flavonoides amarelos em frutos de espécies de pitaias comerciais e nativas do cerrado. Revista Brasileira de Fruticultura, Jaboticabal, v. 35, n. 2, p. 565-570, 2013.

MALAVOLTA, E.; VITTI, G. C.; OLIVEIRA, S. A. Avaliação do estado nutricional das plantas: princípios e aplicações. 2. ed. Piracicaba: Potafós, 1997. $319 \mathrm{p}$.

MARQUES, V. B.; MOREIRA, R. A.; RAMOS, J. D.; ARAÚJO, N. A.; SILVA, F. O. R. Fenologia reprodutiva de pitaia-vermelha no município de Lavras-MG. Ciência Rural, Santa Maria, v.41, n.6, p.984-987, 2011.

MOREIRA, R. A.; RAMOS, J. D.; ARAÚJO, N. A.; MARQUES, V. B. Produção e qualidade de frutos de Pitaia-vermelha com adubação orgânica e granulado bioclástico . Revista Brasileira de Fruticultura, Jaboticabal, v.33,n. 1, p.762-766, 2011. Número especial.
NATALE, W.; PRADO, R. M.; MÔRO, F. V. Alterações anatômicas induzidas pelo cálcio na parede celular de frutos de goiabeira. Pesquisa Agropecuária Brasileira, Brasília, v. 40, n.12, p.1239-1242, 2005.

NELSON, N. A. A photometric adaptation of Somogy method for the determination of glucose. The Journal of Biological Chemistry, Baltimore, v. 153 , n. 2, p. 375-380, 1944.

ORTIZ-HERNÁNDEZ, H. Y. D.; LIVERA M. M.; COLINAS, L. M. T.B.; CARRILLO, S. J .A. Estrés hídrico e intercambio de $\mathrm{CO}_{2}$ de la pitahaya (Hylocereus undatus). Agrociência, Montecillo, v. 33, n. 4 p. 397-405, 1999.

PHEBE, D.; CHEW, M. K.; SURAINI, A. A.; LAI, O. M.; JANNA, O. A. Red-fleshed pitaya (Hylocereus polyrhizus) fruit colour and betacyanin content depend on maturity. International Food Research Journal, Selangor, v. 16, n. 1, p. 233242, 2009.

SANTOS, M. B.; CARDOSO, R. L.; FONSECA, A. A. O. ; CONCEIÇÃO, M. N. Caracterização e qualidade de frutos de umbu-cajá (Spondias tuberosa X S. mombin) provenientes do recôncavo sul da Bahia. Revista Brasileira de Fruticultura, Jaboticabal, v. 32, n. 4, p. 1089-1097, 2010.

SILVA, J. A. A.; GRIZOTTO, R. K.; MIGUEL, F. B.; BÁRBARO, I. M. Caracterização físico-química de frutos de clones de doviális (Dovyalis abyssinica warb), Revista Brasileira de Fruticultura, Jaboticabal, v.33, n.1, p.466-472, 2011. Número especial.

WALL, M. M.; KHAN, S. A. Postharvest quality of dragon fruit (Hylocereus spp.) after X-ray irradiation quarantine treatment. HortScience, Alexandria, v. 43, p. 2115-2119, 2008.

YAH, A. R. C.; PEREIRA, S. S.; VELOZ, C. S.; SAÑUDO, R. B.; DUCH, E. S. Cambios físicos, químicos y sensoriales en frutos de pitahaya (Hylocereus undatus) durante su desarrollo. Revista Fitotecnia Mexicana, Chapingo, v. 31, n. 1, p. 1-5, 2008. 